\title{
A Novel Approach for a Two Area Load Frequency Control in a Competitive Electricity Market Adopting Unscheduled Interchange Price
}

\author{
Murugesan Bhavani', Kamatchi Selvi' ${ }^{2}$, Ravi Durga ${ }^{2}$ \\ ${ }^{1}$ Department of Electrical and Electronics Engineering, Anna University Regional Campus Madurai, \\ Madurai, India \\ ${ }^{2}$ Department of Electrical and Electronics Engineering, Thiagarajar College of Engineering, Madurai, India \\ Email: mbeee@autmdu.ac.in, kseee@tce.edu, linnet.durga@gmail.com
}

Received 21 March 2016; accepted 27 May 2016; published 30 May 2016

Copyright (C) 2016 by authors and Scientific Research Publishing Inc.

This work is licensed under the Creative Commons Attribution International License (CC BY). http://creativecommons.org/licenses/by/4.0/

(c) (†) Open Access

\section{Abstract}

This paper proposes a novel price based load frequency control scheme for a two area system, using an Unscheduled Interchange (UI) price signal, which is ideal and suitable for electricity market. The Government of India has introduced Availability Based Tariff (ABT) structure with an intention of ensuring grid security and to regulate grid indiscipline through Central Electricity Regulatory Commission Regulation. After the introduction of this regulation, the over or under injection by the generating companies (GENCOs) during off peak or peak hours are demoralized and the frequency is maintained at the nominal value. In this paper, the GENCOs instead of reacting to this price signal manually, an automated mode of frequency control is deployed in each area using UI price signal to achieve fast response to load change. The Distribution Company (DISCO) Participation Matrix (DPM) has also been employed in this work to relate the scenario under deregulation of electricity market. The proposed scheme has been verified for different cases by simulating it on a two area system, each having four GENCOs and one DISCO in MATLAB/SIMULINK environment. From the results, it is clearly observed that if the proposed method is employed by all GENCOs, it will certainly enhance the control of system frequency and at the same time throw down the UI liability of market participants. It also ensures that the GENCOs and DISCOs strictly adhere to the bilateral contract following the DISCO participation matrix.

\section{Keywords}

Deregulated Electricity Market, Bilateral Contract, Unscheduled Interchange, Contract 


\section{Participation Factor, DISCO Participation Matrix, Generation Control Error}

\section{Introduction}

After the deregulation of electricity market, the vertical integrated utility has been transformed to novel and divergent entities. Some of them are Generation Companies (GENCOs), Distribution Companies (DISCOs), Transmission Companies (TRANSCOs) and Independent System Operator (ISO). The duty of Independent System Operator is to control all the ancillary services and supervise the transactions that are carried out by the GENCOs and DISCOs. Load Frequency Control is one among such ancillary services. The AGC problem in deregulated electricity market is addressed first in [1] in which the generators are automatically controlled in a system with HVDC connections. The main objective of unbundling electricity market is to fix a price for electricity according to the demand and the generation conditions prevailing at that time. Under deregulated scheme, all the GENCOs and DISCOs in various areas can sign the contract for power transaction and transfer from any GENCO to any DISCO which is known as bilateral transaction [2]. In this paper, the interpretation of DISCO participation matrix for showing bilateral contracts between two areas, as developed in [3] is utilized. In [4], optimization of integral controller gains with Particle Swarm Optimization and Adaptive-Neuro Fuzzy Inference System for LFC problem in deregulated environment is illustrated.

From [5], it is clear that all the generators are able to pick up to 5\% of its capacity instantaneously to meet or manage the load change. In order to improve grid security and to regulate grid discipline, a new tariff system known as Availability Based Tariff (ABT) has been introduced in India. This Availability Based Tariff addresses three kinds of charges namely capacity charge, energy charge and Unscheduled Interchange (UI) charge. Of these, UI charge is the resultant of unscheduled interchange of power. This unscheduled interchange charge acts as a powerful tool for regulating grid frequency. To automate the impact of UI charge in the load frequency control, instead of area control error, generation control error is used in the secondary control loop. The algorithm by which the generation control error is calculated is dealt in [6].

In general, if the GCE (Generation Control Error) is positive, it means that the generation has to be increased in order that more profit is gained. If the GCE is negative, the intention of gaining more profit can be achieved by decreasing the generation. In general, the profit is accrued by the GENCOs if they respond pertinently to the GCE signals. From [7], it is observed that by automating the UI price in load frequency control instead of each generator responding to the UI price manually, the time lapse for the generators to respond is eliminated. During pre-ABT periods, the generators were not paid incentives for producing extra power under peak load conditions, and at the same time, the generators enjoyed some financial advantages without reducing the generation according to the demand during off-peak hours. This will affect the regulation in grid discipline. These drawbacks are overcome by the introduction of Availability Based Tariff. Hence this acts as a potential way to resolve the problem of regulating grid frequency automatically while introducing the deregulated scenario simultaneously. The UI charge makes the DISCOs to contribute for the frequency regulation by increasing the cost of buying power when there is under frequency, and by decreasing the cost of buying power when there is abundant power available. Hence the market participants will get benefited if the grid frequency is under control [8]. Though the incorporation of UI mechanism is time consuming compared to conventional AGC, it can be used as a tool to overcome the flaws in grid discipline in the Indian power market [9]. Several new solutions and technical challenges while implementing AGC in the emerging power market scenario are elaborated in [10].

In view of the above findings, a research gap is identified that so far no trial has been made to consider unscheduled interchange price in frequency control of two area deregulated power system. Hence, in this work an attempt has been made to incorporate the UI mechanism for a two area system, in such a way that the tie-line power flow is regulated, based on the bilateral contracts paving the way for elimination of congestion taking place in deregulated electricity market.

The rest of the paper is structured as follows: Section 2 discusses deregulated electricity market and a brief insight into Availability Based Tariff; Section 3 explains the implementation of proposed work with suitable block diagrams; Section 4 discusses the note-worthy results; and Section 5 gives the imperative conclusions from this work. 


\section{Deregulated Electricity Market}

In deregulated electricity market, all the GENCOs and DISCOs submit their bids as their desired amount for power transaction. The supply and demand curves are drawn based on the bids submitted by the GENCOs and DISCOs. The point at which the demand and supply curve intersects is taken as the Market Clearing Price. Here the Market Clearing Price is taken as the System Marginal Cost. In this work, an assumption is made that the system marginal cost is decided by the supply and demand curves and the generation of scheduled power at each area is based on the system marginal cost which is deemed to be the incremental fuel cost. The simulation model is tested for various system marginal costs. DISCO participation matrix (DPM) is taken as an index for the contract signed between each GENCO and DISCO. The order of DPM indicates the number of GENCOs and DISCOs. The sum of elements in each column should be equal to 1 i.e. each element in the column named as contract participation factor (cpf) is the fraction of power demand of DISCO contracted to be satisfied by the particular GENCO. In this paper, a two area system with four GENCOs and a DISCO in each area is chosen as shown in Figure 1.

The DPM for this two area system is given by Equation (1)

$$
D P M=\left[\begin{array}{ll}
c p f_{11} & c p f_{12} \\
c p f_{21} & c p f_{22} \\
c p f_{31} & c p f_{32} \\
c p f_{41} & c p f_{42} \\
c p f_{51} & c p f_{52} \\
c p f_{61} & c p f_{62} \\
c p f_{71} & c p f_{72} \\
c p f_{81} & c p f_{82}
\end{array}\right]
$$

There are certain cases in which the demand contracted by the DISCO faces changes i.e. there is contract violation; it will be reflected in the area corresponding to DISCO. The proportion by which the GENCOs contribute at the particular area depends on the Area Control Error Participation Factor (apf) which means that area control error due to the local demand change is shared proportionally to all the GENCOs in that area. An assumption is made that at least one GENCO in each area should participate in automatic generation control i.e. it has at least one non-zero area control error participation factor.

Under the present tariff system in India, there is no subdivision of tariff against scheduled and unscheduled interchange of power. Under Availability Based Tariff framed for India, there are separate tariff for scheduled and unscheduled interchange of power. By this splitting mechanism, power trading is promoted. The frequency relation with the UI charge is taken from the Central Electricity Regulatory Commission (CERC) framework, 2012. As per this framework, if the frequency is very low i.e. below $49.5 \mathrm{~Hz}$, then UI charge is very high i.e. the buying entity is charged at a very high price which indirectly forces them to reduce their consumption resulting in enhancement of frequency. If the frequency is very high i.e. above $50.2 \mathrm{~Hz}$, the buying entity is not charged

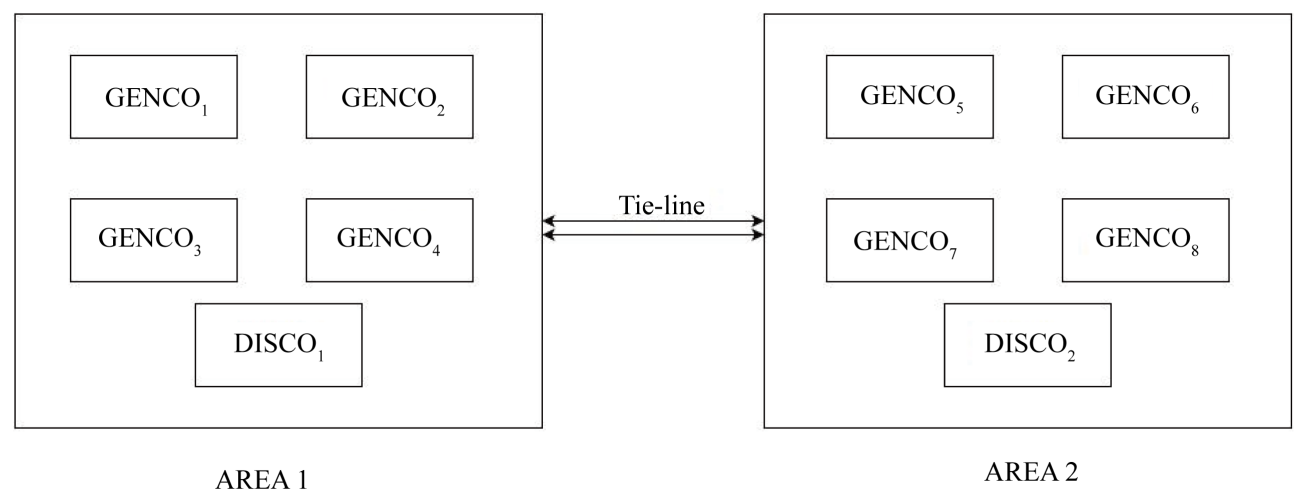

Figure 1. Representation of two area power system in a deregulated electricity market. 
for unscheduled interchange resulting in pulling down the frequency closer to its nominal value. From Figure 2 it is seen that the UI price is inversely proportional to the frequency.

\section{Block Diagram of the Proposed System}

In traditional automatic generation control, tie-line power deviation and steady state frequency deviation are taken as the feedback to cut down the area control error to zero. But in the proposed system, UI price signal is derived from the frequency signals which again is compared with the cost for actual generation.

The signal thus generated is known as Generation Control Error (GCE). This GCE along with the tie-line power deviation is taken as the feedback to reduce the area control error to zero. The comparison of UI price with the actual generation cost is based on the modified Generation Control Error algorithm [7].

This generation control error is used as the input to the integral controller in the secondary control loop of the load frequency control. The generation in each GENCO in all the areas is based on the DISCO participation matrix. The output of each generator is controlled to produce power as per the contract made. This is done using the local economic dispatch controller which receives the signals from the global economic dispatch controller.

The price based load frequency control loop of an area with DPM in deregulated environment is shown in block diagram in Figure 3. The proposed model is tested for a two area system having four GENCOs and one DISCO each. The detailed schematic of price based frequency regulation model for a single area is depicted in Figure 4.

The UI charge is calculated based on the frequency range using Equations (2)-(8) as follows:

Let $S(f)$ be the frequency signal and $S(\rho)$ be the UI price signal,

$$
S(f)=f_{o}+\Delta f
$$

If $S(f)>50.2 \mathrm{~Hz}$

$$
S(\rho)=0 \mathrm{INR} / \mathrm{MWh}
$$

If $50 \mathrm{~Hz}<S(f) \leq 50.2 \mathrm{~Hz}$

$$
S(\rho)=8250 \times(50.2-s(f)) \mathrm{INR} / \mathrm{MWh}
$$

If $49.8 \mathrm{~Hz}<S(f) \leq 50 \mathrm{~Hz}$

$$
S(\rho)=1650+14250 \times(50-S(f)) \mathrm{INR} / \mathrm{MWh}
$$

If $49.48<S(f) \leq 49.8 \mathrm{~Hz}$

$$
S(\rho)=4500+14062.5 \times(49.8-S(f)) \mathrm{INR} / \mathrm{MWh}
$$

If $S(f) \leq 49.48 \mathrm{~Hz}$

$$
S(\rho)=9000 \mathrm{INR} / \mathrm{MWh}
$$

Let $S(\gamma)$ be the incremental fuel cost given by Equation (8),

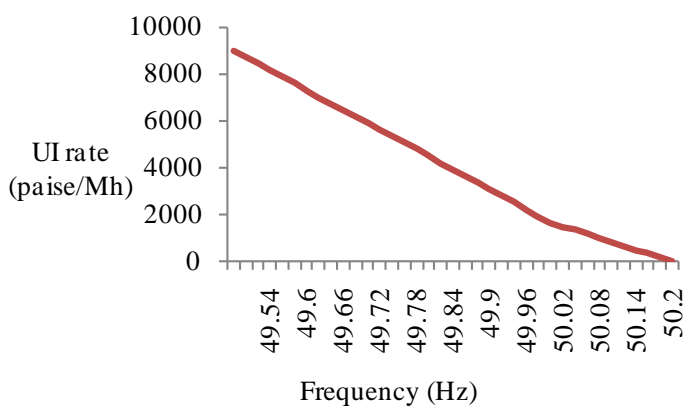

Figure 2. UI charge (Rs/MWh) vs frequency $(\mathrm{Hz})$ as per CERC 2012 regulation. 


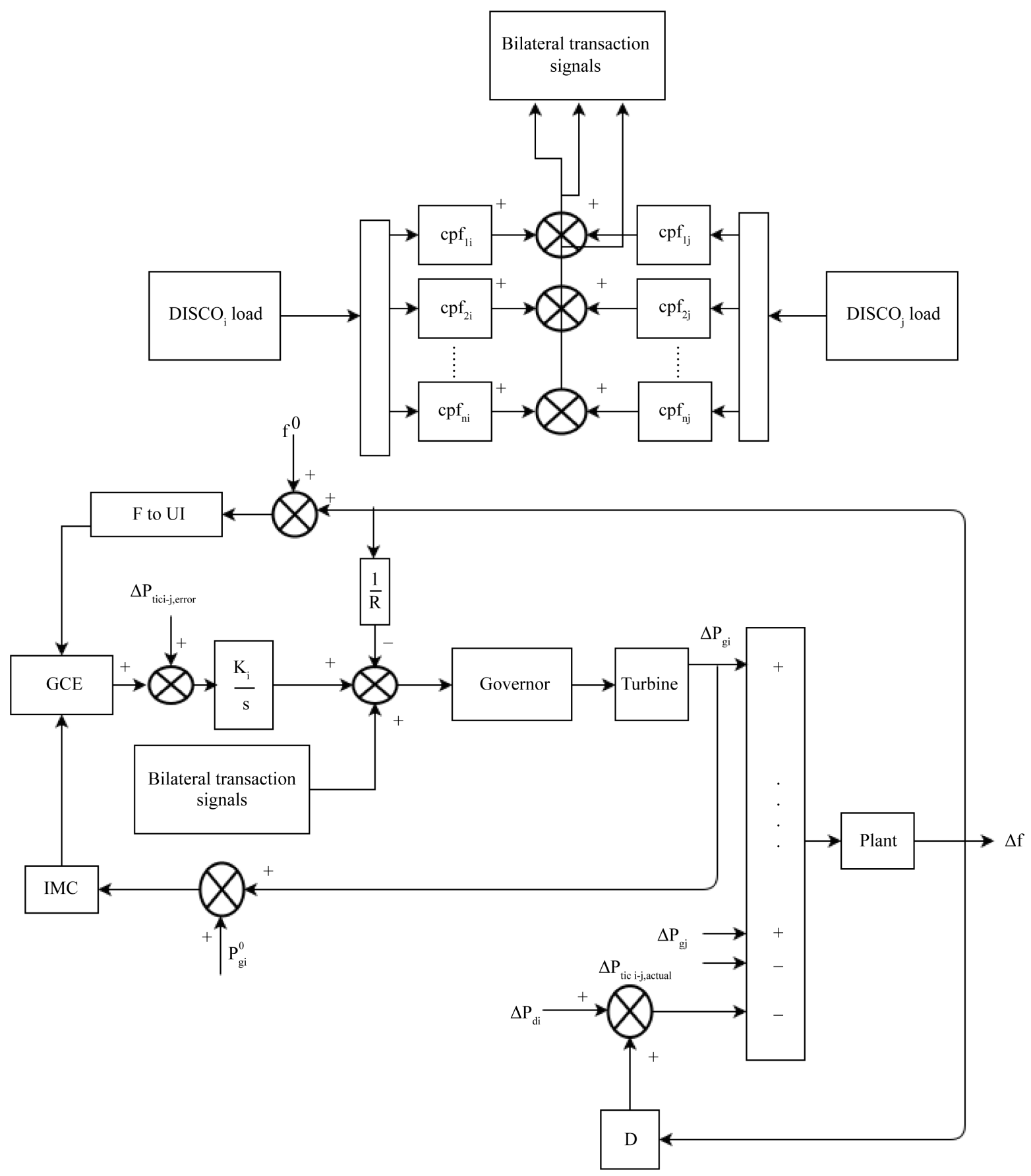

Figure 3. Block diagram of a price based load frequency control of an area $i$ in a deregulated environment.

$$
S(\gamma)=2 \times a_{i} \times S\left(P_{g}\right)+b_{i} \mathrm{INR} / \mathrm{MWh}
$$

where $a_{i}$ and $b_{i}$ are the incremental cost co-efficients.

$S\left(P_{g}\right)$ is given by Equation (9)

$$
S\left(P_{g}\right)=P_{g 0}+\Delta P_{g}
$$

where $P_{g 0}$ is the initial scheduled power and $\Delta P_{g}$ is the load shared by the generator.

The generation control error is calculated based on the Generation Control Error Algorithm which is given in 


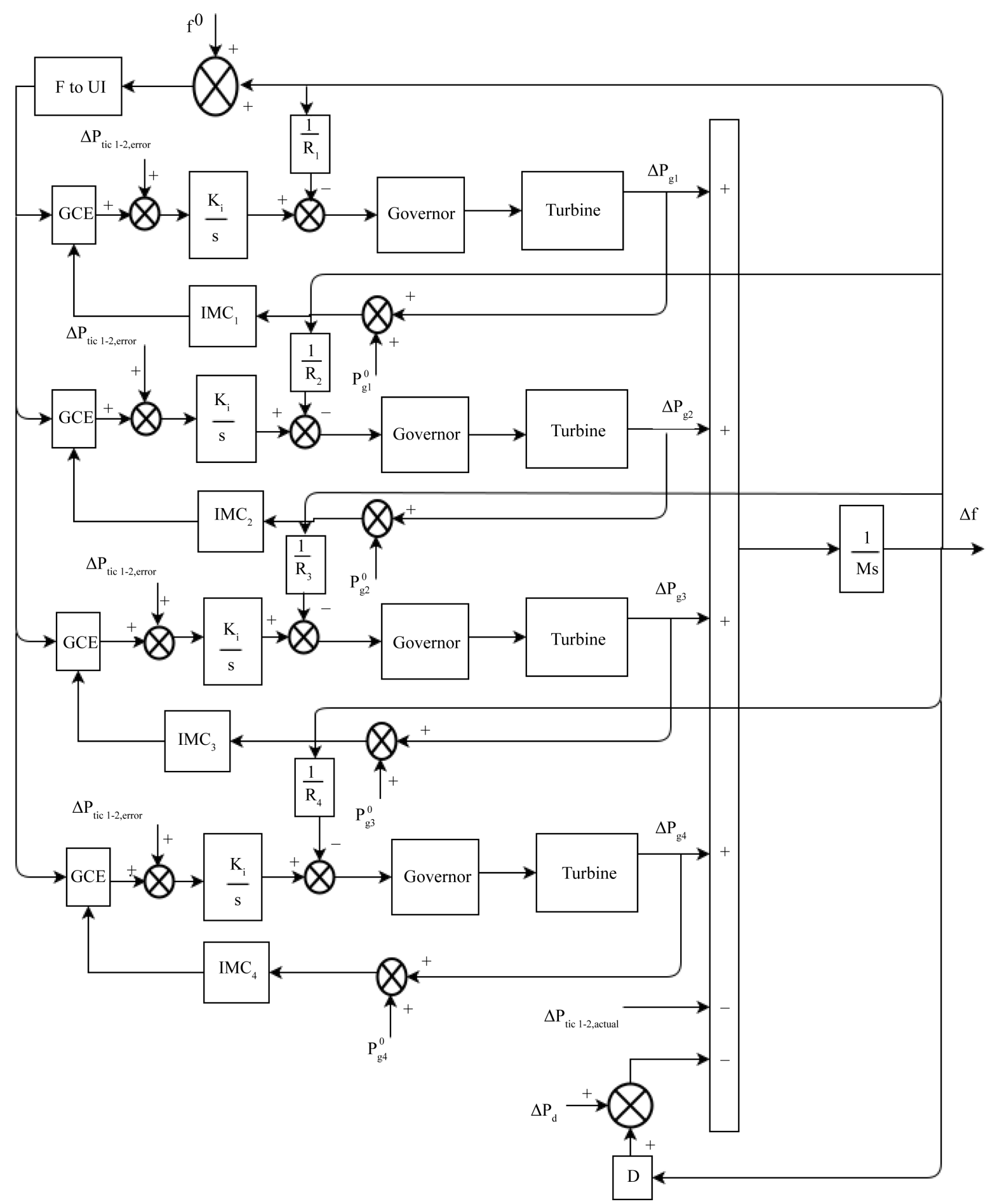

Figure 4. Schematic model of area i with four GENCOs with price based load frequency regulation.

Figure 5.

\section{Results and Discussions}

A power system with two areas is considered to demonstrate the effectiveness of the proposed price based load frequency regulation. The price based secondary frequency control loop contains an integral controller for each 


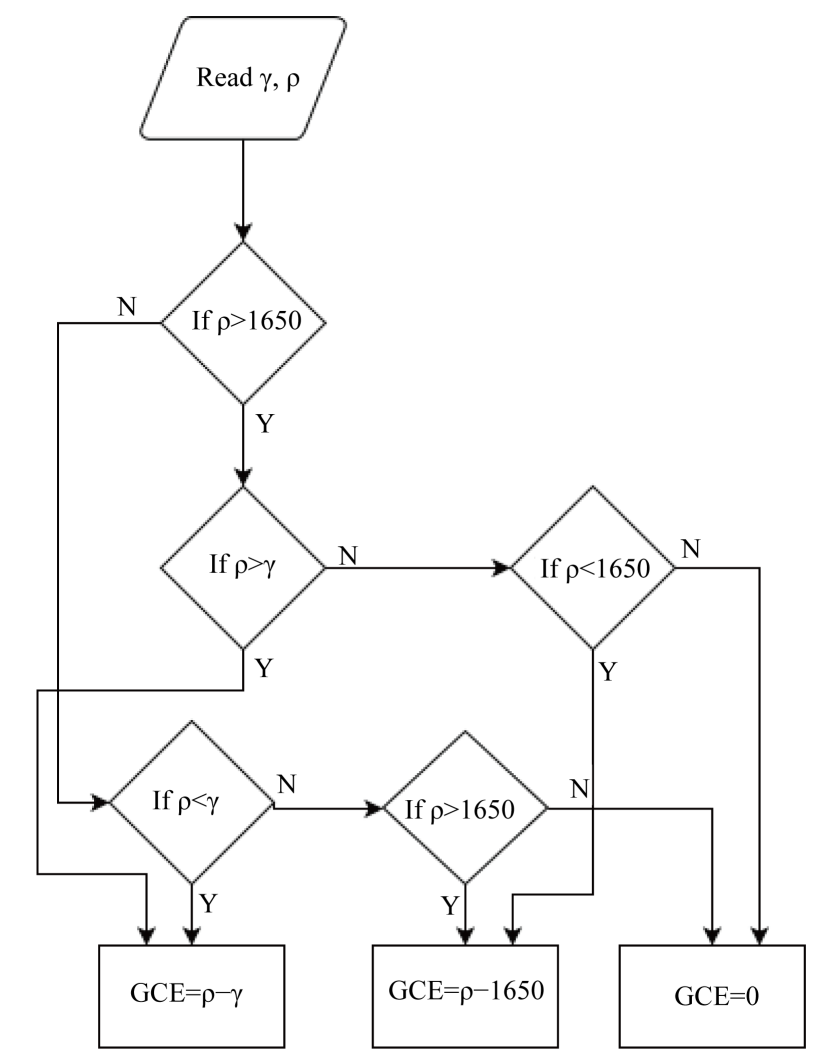

Figure 5. Flowchart for generation control error calculation.

GENCO and is tuned using MATLAB PID tuner. The Automatic PID Tuner allows achieving a good balance between performance and robustness of the integral controller. It is assumed that the two areas are identical with each 5000 MW capacity. The parameters of the test system are given in Tables A1-A3. To validate the proposed method, two test cases are considered. In the first case, it is assumed that the DISCOs in both the areas have load variation of 100 MW each. The load variations of the DISCOs are met by the GENCOs in both the areas based on the bilateral contract executed between them. In the second case, it is assumed that along with the $100 \mathrm{MW}$ load variations of DISCO $_{1}$ and DISCO $_{2}$ there is an additional local load change of $100 \mathrm{MW}$ in DISCO $_{1}$.

Case I: In this case, all the power dispersing authorities will enter into a contract with the power producing authorities for power based on the following DPM

$$
D P M=\left[\begin{array}{cc}
0 & 0 \\
0.25 & 0.15 \\
0.20 & 0.15 \\
0.05 & 0 \\
0 & 0 \\
0.25 & 0.3 \\
0.20 & 0.3 \\
0.05 & 0.1
\end{array}\right]
$$

The simulation results for this case are shown in Figure 6. When the load of $\mathrm{DISCO}_{1}$ and $\mathrm{DISCO}_{2}$ increase their demand by $100 \mathrm{MW}$, the system frequency rapidly drops from the nominal value. The GENCOS in the two areas will respond to this load variation based on the DISCO participation matrix (DPM) and UI price signal, this co-action drives the system frequency to settle closer to the nominal value. From Figure 6(a), it is evident that the frequency deviation drops suddenly and finally settle to a value around $49.99 \mathrm{~Hz}$. It leads the UI price of both area I and II to settle at 1732 Rs/MW has depicted in Figure 6(b). 


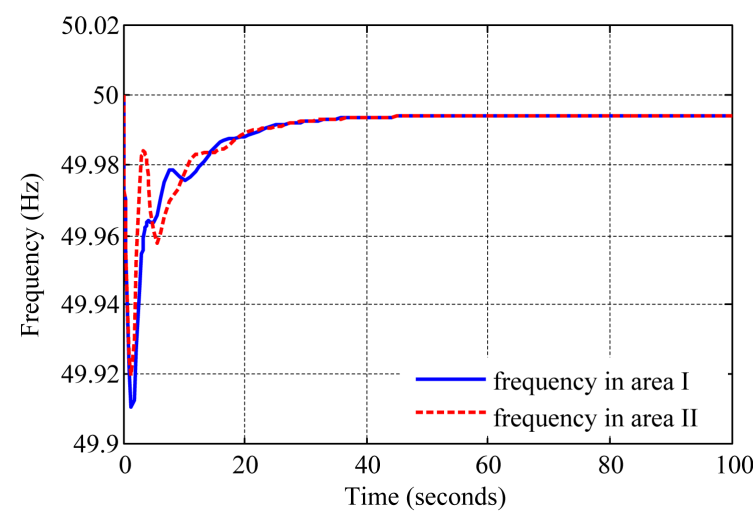

(a)

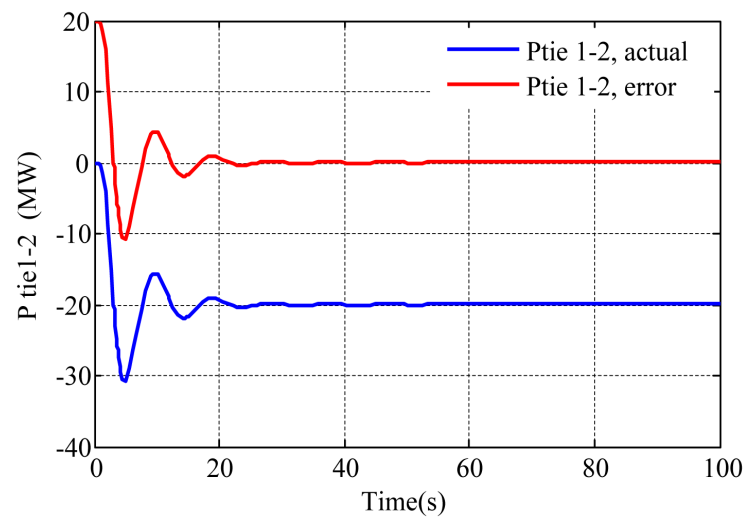

(c)

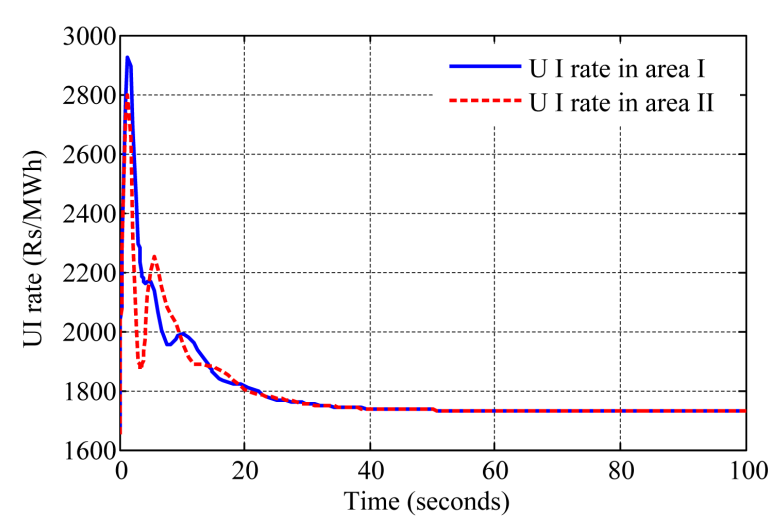

(b)
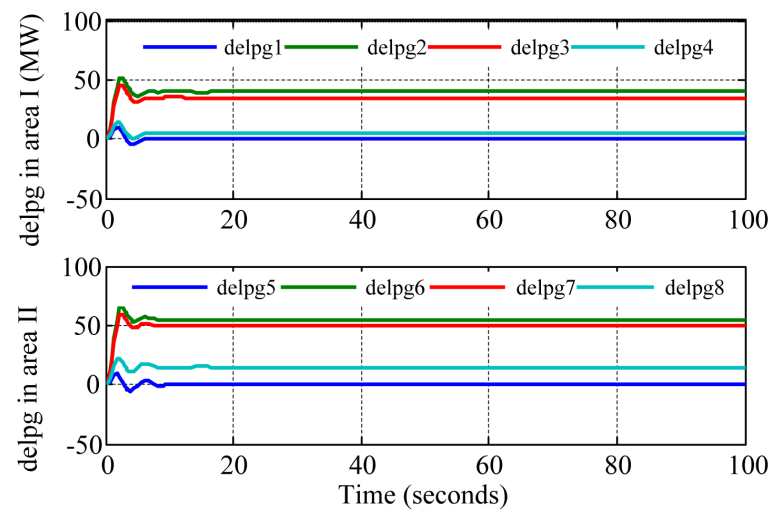

(d)

Figure 6. (a) System frequency (Hz); (b) UI rate (Rs/MWhr); (c) Change in tie line power, actual and error (MW); (d) change in generation in each unit in area I and II (MW).

The scheduled tie-line power deviation is as given by,

$$
\begin{aligned}
\Delta P_{\text {tie1-2,scheduled }}= & \text { Demand of DISCOs in area II from GENCOs in area I } \\
& - \text { Demand of DISCOs in area I from GENCOs in area II } \\
= & ((0+0.15+0.15+0) \times 0.1)+((0+0.25+0.2+0.05) \times 0.1) \text { p.u.MW } \\
= & -0.02 \text { p.u.MW } \\
= & -20 \mathrm{MW}
\end{aligned}
$$

From Figure 6(c), it is observed that, as the change in tie-line power flow error settles to zero, the change in actual tie-line power flow from area 2 to area 1matches the scheduled value of $20 \mathrm{MW}$.

Since $\mathrm{GENCO}_{1}$ and $\mathrm{GENCO}_{5}$ are already generating power at their maximum capacity, they cannot contribute for any load change. Hence the corresponding cpfs are zero in the DPM matrix. The change in generation of GENCO2 is given by

$$
\begin{aligned}
\Delta P_{g 2} & =\left(c p f_{21} \times \Delta P_{L 1}\right)+\left(c p f_{22} \times \Delta P_{L 2}\right) \\
& =(0.25 \times 0.1)+(0.15 \times 0.1)=0.04 \text { p.u.MW }=40 \mathrm{MW}
\end{aligned}
$$

Similarly $\Delta P_{g 3}=0.035$ p.u.MW $=35 \mathrm{MW}, \Delta P_{g 4}=0.005$ p.u.MW $=5 \mathrm{MW}, \Delta P_{g 6}=0.055$ p.u. $\mathrm{MW}=55 \mathrm{MW}$, $\Delta P_{g 7}=0.05$ p.u.MW $=50 \mathrm{MW}, \Delta P_{g 8}=0.015$ p.u.MW $=15 \mathrm{MW}$. The total change in generation by GENCOs in the system sums to a value of $200 \mathrm{MW}$ i.e. the total load change considered in this case.

From Figure 6(d), the actual power generated by each GENCO matches the desired value as per the DPM.

Case II: There is a possibility that the DISCO in a deregulated electricity market may violate the agreement 
by drawing excess power than that stipulated in the agreement. This local demand should be satisfied by the power producing authorities in the corresponding DISCO area. The change in demand and the DPM is assumed to be similar with case I. In addition to this, it is assumed that there is a contract violation of $100 \mathrm{MW}$ by DISCO $_{1}$. Simulation results are shown in Figures 7(a)-(d). Though the DPM and the change in demand are same for both the cases, due to this contract violation, the trajectory of change in generation of area 1 is not identical to that of case I. GENCOs in area 1 share this contract violation based on the Area Control Error Participation Factor (apf). The values of apf are chosen as apf $\operatorname{ap}_{11}=0, \operatorname{apf}_{21}=0.25, \operatorname{apf}_{31}=0.25, \operatorname{apf}_{41}=0.5$, apf $_{52}=0$, $\operatorname{apf}_{62}=0.25, \operatorname{apf}_{72}=0.25$, apf $_{82}=0.5$. The system frequency of area1 abruptly drops to a value of about $49.8 \mathrm{~Hz}$ due to contract violation and settles by the corrective action taken by the price based feedback in the secondary loop of frequency control. From the simulation result it is evident that due to contract violation in area 1, the load sharing by the GENCOs in area 1 differs in case II with that of case I.

The load shared by GENCO2 is given by

$$
\begin{aligned}
\Delta P_{g 2} & =\left(c p f_{21} \times \Delta P_{L 1}\right)+\left(c p f_{22} \times \Delta P_{L 2}\right)+\left(a p f_{21} \times \text { Local demand in area } 1\right) \\
& =(0.25 \times 0.1)+(0.15 \times 0.1)+(0.25 \times 0.1) \text { p.u.MW } \\
& =0.065 \text { p.u. } \mathrm{MW}=65 \mathrm{MW}
\end{aligned}
$$

Similarly for the remaining GENCOs in area I,

$$
\Delta P_{g 3}=0.06 \text { p.u.MW }=60 \mathrm{MW}, \Delta P_{g 4}=0.055 \text { p.u.MW }=55 \mathrm{MW} .
$$

The change in generation for the units in area I and II are shown in Figure 7(d).

Since there is no contract violation in area II, the load sharing by each GENCO in area II remains the same as in case I. It can be observed from Figure 7(b) that, due to contract violation in area I, the peak value of UI price

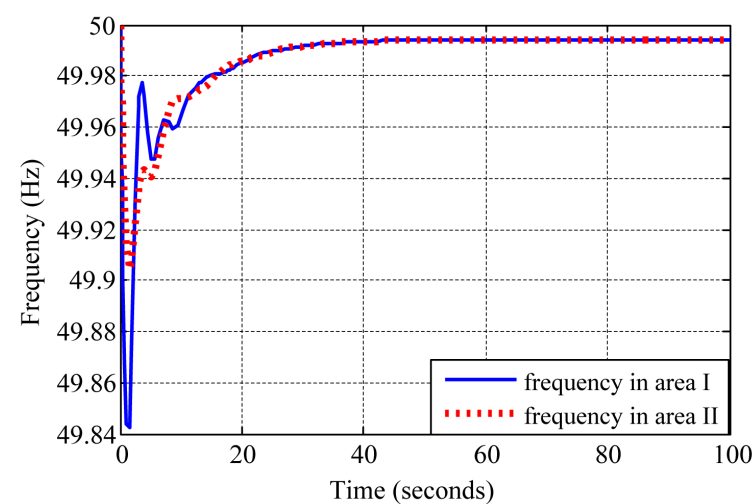

(a)

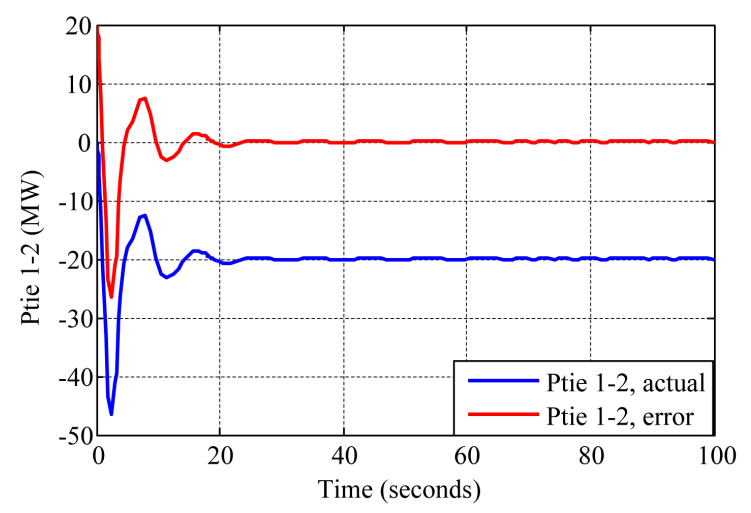

(c)

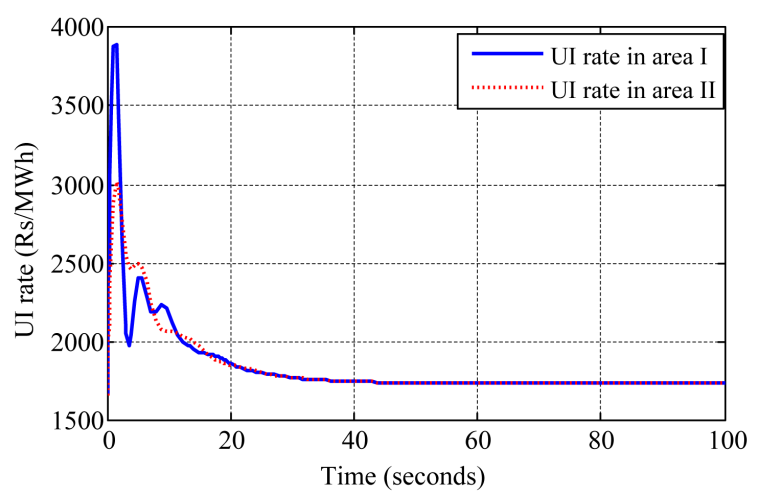

(b)
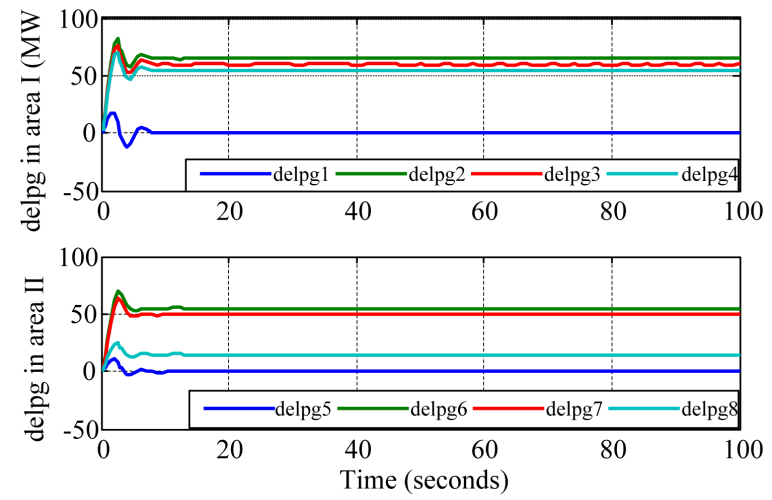

(d)

Figure 7. (a) System frequency (Hz); (b) UI rate (Rs/MWhr); (c) Change in tie line power, actual and error (MW); (d) change in generation in each unit in area I and II (MW). 
Table 1. Change in generation of GENCOs for case I and II.

\begin{tabular}{|c|c|c|c|c|c|c|c|c|}
\hline \multirow{2}{*}{ Case } & \multicolumn{8}{|c|}{ Average change in generation (MW) } \\
\hline & $\Delta \mathrm{P}_{\mathrm{g} 1}$ & $\Delta \mathrm{P}_{\mathrm{g} 2}$ & $\Delta \mathrm{P}_{\mathrm{g} 3}$ & $\Delta \mathrm{P}_{\mathrm{g} 4}$ & $\Delta \mathrm{P}_{\mathrm{g} 5}$ & $\Delta \mathrm{P}_{\mathrm{g} 6}$ & $\Delta \mathrm{P}_{\mathrm{g} 7}$ & $\Delta \mathrm{P}_{\mathrm{g} 8}$ \\
\hline I & 0 & 30.6 & 26.8 & 3.6898 & 0 & 42 & 38 & 11.2 \\
\hline II & 0 & 46.0 & 42.5 & 38.895 & 0 & 40 & 35.5 & 10.6 \\
\hline
\end{tabular}

Table 2. Profit earned by GENCOs for case I and II.

\begin{tabular}{|c|c|c|c|c|c|c|c|c|c|c|}
\hline \multirow{2}{*}{ Case } & \multirow{2}{*}{$\begin{array}{l}\text { Avg. } \\
\text { Frequency } \\
\text { (Hz) }\end{array}$} & \multirow{2}{*}{$\begin{array}{c}\text { Avg. Change } \\
\text { in UI } \\
\text { (Rs/MWh) }\end{array}$} & \multicolumn{8}{|c|}{ Profit earned by each GENCO in area 1 and 2 for 100 seconds (Rs) } \\
\hline & & & GEN 1 & GEN 2 & GEN 3 & GEN4 & GEN 5 & GEN 6 & GEN 7 & GEN8 \\
\hline I & 49.9848 & 1867.3 & 0 & 1589.4 & 1389.8 & 191.3 & 0 & 2143.7 & 1947.1 & 571.9 \\
\hline II & 49.9830 & 1892.8 & 0 & 2419.5 & 2232.8 & 2045 & 0 & 2005.2 & 1822.9 & 547.2 \\
\hline
\end{tabular}

in this area shoots up to 3895 Rs/MWh when compared to the peak value of UI price 3014 Rs/MWhin area II.

In real time, the incentives given and penalties imposed on the participants are based on the average frequency for the time block of 15 minutes. The average change in generation for case I and II for a short time period of 100 seconds is tabulated in Table 1. The profit earned by various GENCOs is given in Table 2. The automated UI mechanism has made the GENCOs to earn profit and at the same time, it indirectly forces the DISCOs to reduce the unscheduled interchange. The GENCOs with high incremental fuel cost, which is not preferred by DISCOs under normal condition, will get an opportunity to serve the load and earn profit during contract violation.

\section{Conclusion}

In this research, load frequency control is performed for a two area deregulated electricity market by incorporating UI mechanism in the secondary loop. With the penetration of distributed generation in electricity market, the power imbalances are inherent leading to variation in system frequency. The UI mechanism adopted here acts as a tool to carry out prominent adjustment of scheduled generated power to match the actual load behavior. The results of load variations for bilateral contract with and without contract violation after incorporating automated UI mechanism prove the competence of the proposed method, since this scheme is capable of quickly balancing the power demand with supply and restore frequency even with contingencies like generation outage. In the meanwhile, the possibility of unscheduled interchange truncates within a shorter time due to the automation of UI signal in load frequency control. The UI liability of power market participants can be immensely reduced. The real time price signal will enable the high cost generating plants with renewable energy sources to afford the local demand changes. In the future, the work can be extended for a real time system with multilateral transactions.

\section{References}

[1] Bakken, B.H. and Grande, O.S. (1998) Automatic Generation Control in a Deregulated Power System. IEEE Transactions on Power Systems, 13, 4.

[2] Ezra, H., Rick, H. and Smith, A. (2008) Bilateral Contracting in Deregulated Electricity Markets. A Report to the American Public Power Association. https://www.publicpower.org/files/PDFs/EMRISynapseBilateralsReport2008.pdf

[3] Vaibhav, D. and Pai, M.A. (2001) Simulation and Optimization in an AGC System after Deregulation. IEEE Transactions on Power Systems, 16, 3. http://dx.doi.org/10.1109/59.932285

[4] Hosseini, S.H. and Etemadi, A.H. (2008) Adaptive Neuro-Fuzzy Inference System Based Automatic Generation Control. Electric Power Systems Research, 78, 1230-1239. http://dx.doi.org/10.1016/j.epsr.2007.10.007

[5] Hassan Bevrani, M. (2014) Robust Power System Frequency Control. Springer International Publishing, Cham.

[6] Parida, S.K., Singh, S.N. and Srivastava, S.C. (2009) An Integrated Approach for Optimal Frequency Regulation Service Procurement in India. Energy Policy, 37, 3020-3034.

[7] Saurabh, C. and Ashwani, K. (2010) A Price Based Automatic Generation Control Using Unscheduled Interchange 
Price Signals in Indian Electricity System. International Journal of Engineering, Science and Technology, 2, 23-30. http://dx.doi.org/10.4314/ijest.v2i2.59135

[8] Pujara, S.M. and Kotwal, C.D. (2014) Optimized Integral Gain Controllers for Price Based Frequency Regulation of Single Area Multi-Unit Power System. International Journal on Electrical Engineering and Informatics, 6, 2.

[9] Gargi, K., Niladri, C. and Kamal Krishna, M. (2014) Unscheduled Interchange (UI) Price Based Secondary Frequency Control of an Interconnected Hybrid Power System. Annual IEEE India Conference (INDICON), Pune, 11-13 December 2014, 1-6.

[10] Soonee, S.K., Narasimhan, S.R. and Pandey, V. (2006) Significance of Unscheduled Interchange Mechanism in the Indian Electricity Supply Industry. International Conference on Power system Operation in Deregulated Regime, Varanasi, 6-7 March 2006, 1-4. 


\section{Appendix A}

Table A1. System data.

\begin{tabular}{ccccc}
\hline Parameters & $\mathrm{G}_{1}$ & $\mathrm{G}_{2}$ & $\mathrm{G}_{3}$ & $\mathrm{G}_{4}$ \\
\hline Capacity(MW) & 1500 & 1500 & 1000 & 1000 \\
b ( INR/MWh) & 800 & 1000 & 1600 & 2000 \\
c (INR/MW' ${ }^{2}$ ) & 0.3 & 0.3 & 0.4 & 0.4 \\
Initial Generation $\mathrm{P}_{\text {go }}$ & 1500 & 1416.66 & 312.5 & 0 \\
\hline
\end{tabular}

Where b and c are the cost coefficients of each generator for calculating the incremental fuel cost.

Table A2. Area parameters.

$\begin{array}{lc}\text { M (MW-s/Hz) } & 1000 \\ \mathrm{D}(\mathrm{MW} / \mathrm{Hz}) & 100 \\ \mathrm{~F}^{0}(\mathrm{~Hz}) & 50 \\ & 0.0707 \\ \text { Power Co-efficient, } \mathrm{T}_{12} \text { (p.u) } & 1850\end{array}$

Where $\mathrm{M}$ is the inertia constant, $\mathrm{D}$ is the damping coefficient.

Table A3. Droop, governor and turbine time constant.

\begin{tabular}{ccccc}
\hline Parameters & Machine 1 & Machine 2 & Machine 3 & Machine 4 \\
\hline Droop R & $6 \%$ & $6 \%$ & $6 \%$ & $6 \%$ \\
Governor time constant $\mathrm{T}_{\mathrm{sg}}(\mathrm{sec})$ & 0.3 & 0.3 & 0.3 & 0.3 \\
Turbine time constant $\mathrm{T}_{\mathrm{t}}(\mathrm{sec})$ & 0.5 & 0.5 & 0.5 & 0.5 \\
\hline
\end{tabular}

